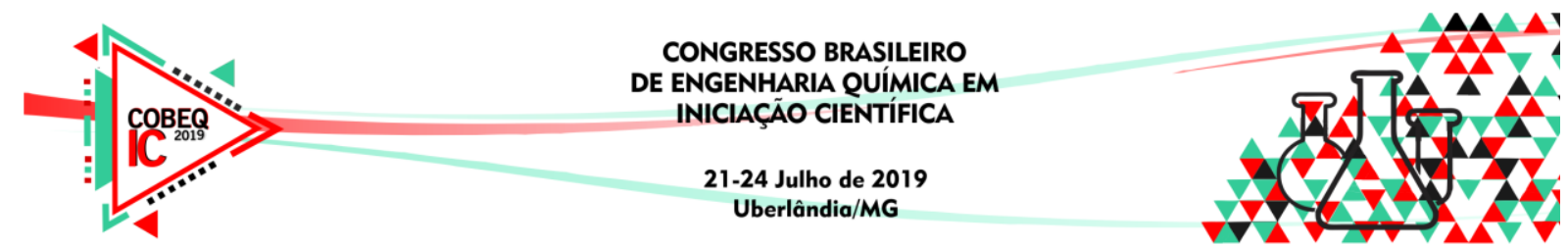

\title{
A CINÉTICA DE SECAGEM E A CONTRAÇÃO VOLUMÉTRICA DE FRUTOS DE ARAÇÁ (Psidium cattleianum) DESIDRATADOS EM SECADOR DE BANDEJAS
}

\author{
P. S. MAGALHÃES ${ }^{1}$ e A. O. ROCHA ${ }^{1}$, R. C. NEPOMUCENO ${ }^{1}$ \\ ${ }^{1}$ Universidade Patos de Minas, Departamento de Engenharia Química \\ E-mail para contato: patriciasilva@ unipam.edu.br
}

\begin{abstract}
RESUMO - O presente trabalho tem como objetivo realizar a desidratação do araçá, levando-se em consideração aspectos cinéticos e de contração volumétrica. Os experimentos foram conduzidos em secador de bandeja nas temperaturas de $50,60,70$ e $80^{\circ} \mathrm{C}$, sendo o teor de água acompanhado por método gravimétrico. Foram adotados como objeto de estudo os modelos cinéticos: Midilli et al. (2002) et al., Newton e Lewis, Henderson e Pabis e Page. Para determinação da contração volumétrica os frutos foram considerados como elipsoides, sendo que durante a secagem três amostras tiveram suas dimensões lineares medidas por paquímetro digital. O modelo de cinética de secagem de Midilli et al.(2002), apresentou em média $99,9 \%$ de representatividade entre os dados observados e estimados. O modelo de Bala \& Woods adaptado se mostrou satisfatório na predição da contração volumétrica, com coeficiente de determinação $\left(\mathrm{R}^{2}\right)$ de 0,971 .
\end{abstract}

\section{INTRODUÇÃO}

$\mathrm{O}$ araçazeiro possui frutos que fornecem maior variedade à dieta, através de alimentos nutritivos ricos em compostos funcionais que poderiam atuar como antioxidantes naturais, trazendo proteção ao organismo. O araçá possui benefícios ao organismo, com atividades antimicrobiana, antioxidante, anti-inflamatória, antifúngica, cardioprotetora, antimutagênica, antiproliferativa, hipoglicemiante, hipolipidêmica, antipirética, antidiarreica, antibacteriana, diurética, hepatoprotetora, nefroprotetora, antiálergica, antigenotóxica e antiúlcera. Os frutos são especialmente ricos em minerais como cálcio, fósforo e ferro e elementos funcionais, tais como vitaminas A, B e C e compostos fenólicos com ação antioxidante (CUNHA, 2014).

Segundo D'Andres (2015) o elevado teor de umidade desse fruto é o principal responsável pelo desenvolvimento de bactérias, fungos e leveduras. Visando a sua conservação, sem perdas significativas nutricionais, a secagem é um dos métodos mais utilizados industrialmente. Esta por sua vez, se caracteriza pelo movimento da água do interior do produto até a superfície externa do fruto, envolvendo diversos mecanismos de transferência de calor e massa. A secagem colabora no prolongamento da qualidade do produto estendendo o período de armazenamento e promovendo o aumento de sua vida útil. Este trabalho tem como objetivo apresentar um estudo sobre a cinética de secagem e a contração volumétrica do araçá, verificando através de modelos matemáticos teóricos o comportamento durante a secagem. 


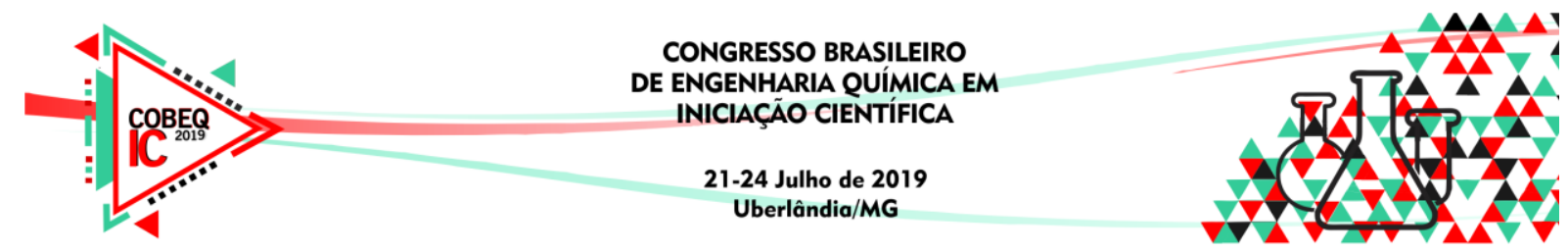

\section{MATERIAIS E MÉTODOS}

\subsection{Matéria-prima e caracterização física}

Os frutos foram coletados no município de Brasilândia de Minas (530 $\mathrm{m}$ de altitude, $17^{\circ}$ $00^{\prime} 35^{\prime \prime} \mathrm{S} / 46^{\circ} 00^{\prime} 34^{\prime \prime O}$ ). Após a coleta os frutos permaneceram congelados até a realização das análises as quais foram desenvolvidas no Laboratório de Engenharia Química do UNIPAM. Antes dos ensaios de secagem a amostra foi descongelada e caracterizada fisicamente por meio de suas dimensões lineares (Figura 1) obtendo-se os valores médios de volume inicial.

Figura 1 - Desenho esquemático de um esferoide tri axial com suas dimensões lineares e frutos de araçá
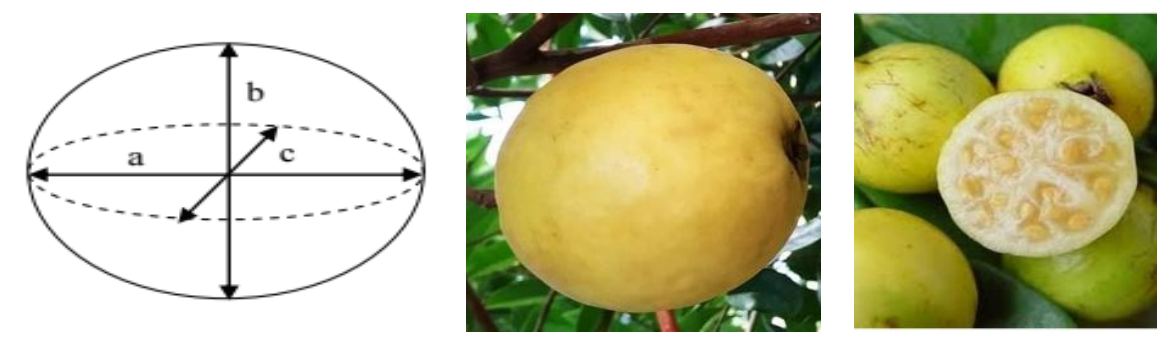

\subsection{Cinética de Secagem}

Os experimentos de secagem foram conduzidos em um desidratador Pardal ${ }^{\circledR}$ PE 60 , com o auxílio de balança Digimed ${ }^{\circledR}$ DG 15 e do paquímetro digital. Os frutos foram cortados ao meio no sentido transversal e dispostos em monocamada na bandeja (devidamente higienizada) com a face circular dos frutos voltada para baixo. Para a secagem foram adotadas as temperaturas de $50,60,70$ e $80^{\circ} \mathrm{C}$, sendo realizadas pesagens em intervalos de tempos consecutivos até obtenção de equilíbrio cinético. Para determinação da cinética de secagem parâmetros como a umidade $(\mathrm{X})$ e taxa de umidade $(\mathrm{R})$ foram calculados com base na massa seca representados nas Equações 1,2 e 3.

$$
\begin{aligned}
& X_{t}=\frac{W-W_{s}}{W_{s}} \\
& X=X_{t}-X^{*} \\
& R=-\frac{L_{s}}{A} \cdot \frac{d X}{d t}
\end{aligned}
$$

Modelos matemáticos são utilizados para a simulação da cinética de secagem que representam a perda de água durante o período de secagem. A Tabela 1 explicita os modelos empíricos e semi-teóricos apresentados na literatura para produtos agrícolas, sendo esses avaliados no presente trabalho. Os modelos se baseiam na razão de umidade (RU), conforme expresso pela Equação 4, calculada pela razão entre a umidade livre no instante t e a umidade livre inicial na amostra. 


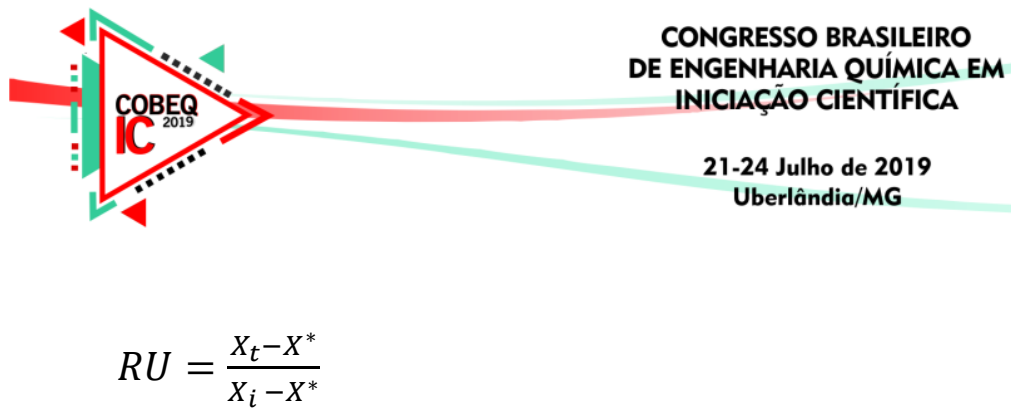

Tabela 1 - Modelos matemáticos para predição da cinética de secagem

\begin{tabular}{|c|c|}
\hline Descrição & Modelos \\
\hline Page & $R U=e^{-k t^{n}}$ \\
\hline Midilli et al. & $R U=a e^{-k t^{n}+b t}$ \\
\hline Henderson e Pabis & $R U=a e^{-k t}$ \\
\hline Newton e Lewis & $R U=e^{-k t}$ \\
\hline
\end{tabular}

a, b, n e k $\left(\min ^{-1}\right)$ : constantes características. Fonte: Corrêa Filho et al. (2014) e Faria et al.(2012).

\subsection{Contração Volumétrica}

De acordo com Resende (2005) a contração volumétrica é o encolhimento dos grãos durante o processo de secagem, provocado pela redução do teor de água no material. Para avaliar o grau de encolhimento, a forma do fruto foi ajustada a um elipsoide (cortado ao meio). Durante a secagem, três frutos com dimensões homogêneas e distintas tiveram seus eixos ortogonais medidos por um paquímetro digital modelo MTX® Digital Caliber, durante os intervalos de pesagens. Para modelar matematicamente o grau de encolhimento dos frutos de araçá durante a secagem foram adotados os modelos empíricos descritos na Tabela 2, utilizando-se a contração volumétrica calculada pela Equação 5, determinada a partir dos volumes inicias e finais correspondente ao tempo (CORRÊA FILHO et al., 2014).

$$
\Psi=\frac{V}{V_{0}}
$$

Tabela 2 - Modelos matemáticos para predição da contração volumétrica

\begin{tabular}{|c|c|}
\hline Descrição & Modelo \\
\hline Linear adaptado & $\Psi=(a+b X) T^{c}$ \\
\hline Polinomial & $\Psi=a+b X+C X^{2}$ \\
\hline Bala \& Woods modificado & $\Psi=1-a\left(1-e^{b\left(X_{i}-X\right)}\right)$ \\
\hline Bala \& Woods adaptado & $\Psi=\left(1-a\left(1-e^{-b\left(X_{i}-X\right)}\right)\right) T^{c}$ \\
\hline
\end{tabular}

a, b, c: constantes características. Fonte: Corrêa Filho et al. (2014).

\section{RESULTADOS E DISCUSSÕES}

\subsection{Cinética de secagem}

A representação do processo de secagem de diversos produtos vem sendo utilizada na tentativa de predizer os fenômenos ocorridos. Celestino (2010) acrescenta que, a rapidez com que o alimento perde umidade é controlada pelas variáveis do processo (temperatura, velocidade e umidade relativa do ar) e pelas características da matriz do alimento. As curvas da cinética de secagem obtidas para o araçá são apresentadas na Figura 2A.

Pela Figura 2A nota-se que a temperatura exerce significativa influência sobre a cinética de secagem do araçá, onde o tempo gasto para atingir o equilíbrio a $50^{\circ} \mathrm{C}$ é cerca de duas 

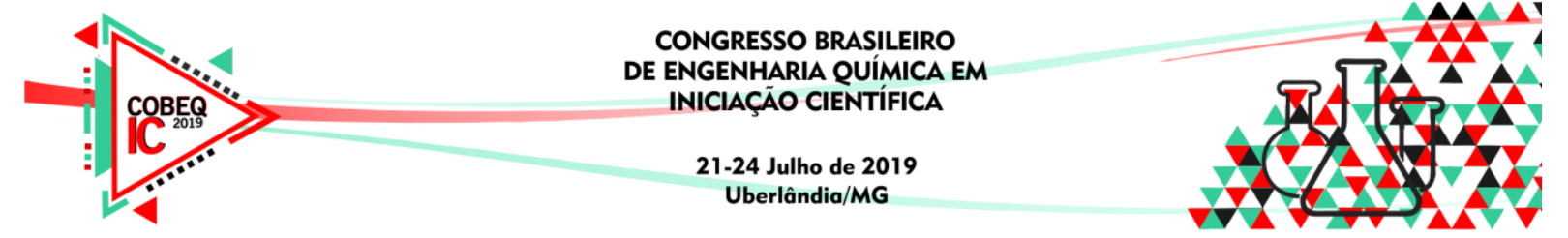

vezes maior que a $80^{\circ} \mathrm{C}$. A Figura $2 \mathrm{~B}$ explicita as taxas de secagem perante a umidade livre. Os resultados obtidos por Fonseca (2017) assemelham-se aos resultados obtidos neste estudo, onde se observam períodos distintos de taxa de secagem. De acordo com Park et al. (2007) no período de taxa de secagem constante a água evapora como água livre, pois sua pressão de vapor é constante e igual à pressão de vapor da água pura na temperatura do produto. Já no período decrescente, a taxa de transferência de umidade é proporcional à diferença instantânea entre o teor de água do produto e o teor de água esperado do material em equilíbrio com o ar de secagem (Brooker et al.,1992).

Figura 2 - (A) Efeitos da temperatura sobre a cinética de secagem de frutos de araçá e a predição pelo modelo cinético de Midilli et al. (2002) et al. e (B) Taxa de secagem
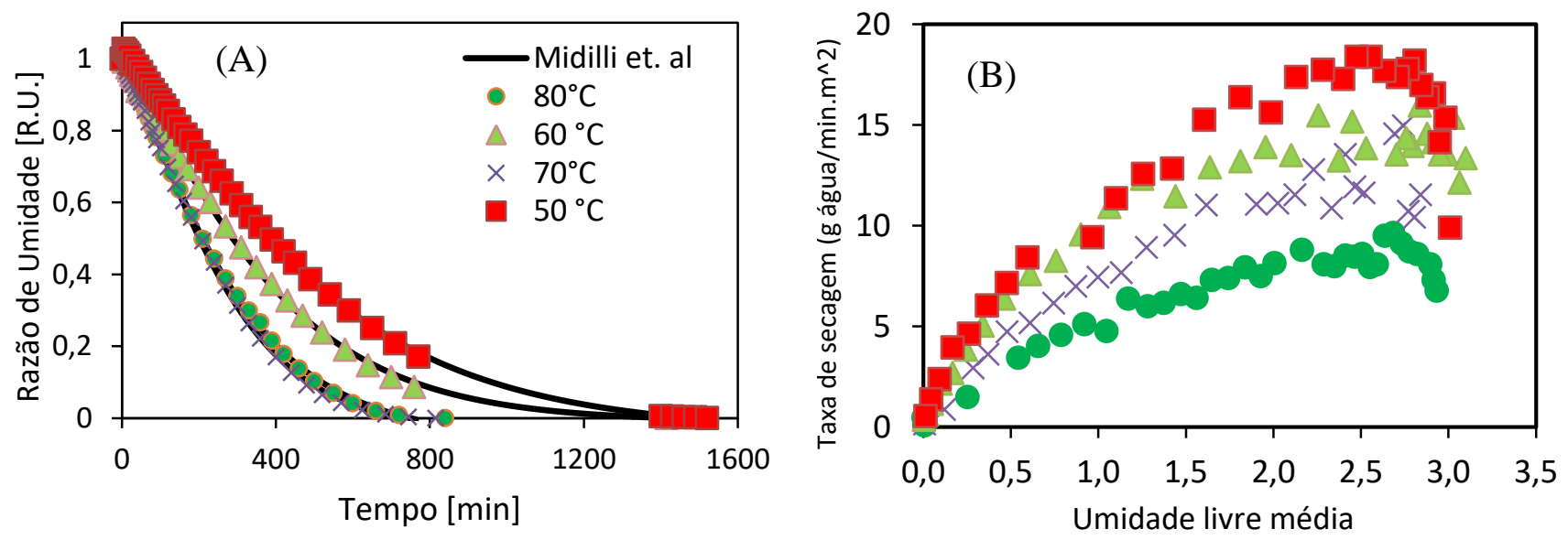

Adotando os modelos cinéticos (Tabela 3) por meio do software SCILAB ${ }^{\circledR}$, versão 5.5.2., obtiveram-se as curvas de cinética de secagem, tendo o modelo matemático de Midilli et al. (2002), apresentado coeficiente de determinação médio de 99,9\% na representatividade entre os dados observados e estimados, sendo assim o mais adequado para predizer o fenômeno de secagem.

Tabela 3 - Parâmetros dos modelos de cinética de secagem para o fruto araçá.

\begin{tabular}{|c|c|c|c|c|c|}
\hline Modelos & Parâmetros & $50^{\circ} \mathrm{C}$ & $60^{\circ} \mathrm{C}$ & $70{ }^{\circ} \mathrm{C}$ & $80{ }^{\circ} \mathrm{C}$ \\
\hline \hline \multirow{4}{*}{ Page } & $k\left(\mathrm{~min}^{-1}\right)$ & 0,0002649 & 0,0007744 & 0,000674 & 0,000748 \\
\cline { 2 - 6 } & $N$ & 1,3227522 & 1,2022838 & 1,311225 & 1,280982 \\
\cline { 2 - 6 } & $R^{2}$ & 0,9989428 & 0,9992474 & 0,998611 & 0,998905 \\
\hline \multirow{4}{*}{ Midilli et al. } & $A$ & 1,0186818 & 0,9863895 & 0,9845395 & 0,9954334 \\
\cline { 2 - 6 } & $k\left(\mathrm{~min}^{-1}\right)$ & 0,0004625 & 0,0006001 & 0,0005886 & 0,0007482 \\
\cline { 2 - 6 } & $N$ & 1,2305975 & 1,2409927 & 1,3240423 & 1,2454621 \\
\cline { 2 - 6 } & $b\left(\mathrm{~min}^{-1}\right)$ & $-1,73 \mathrm{E}-05$ & $-4,90 \mathrm{E}-06$ & $-3,64 \mathrm{E}-05$ & $-5,04 \mathrm{E}-05$ \\
\hline \multirow{3}{*}{ Henderson e Pabis } & 0,9996603 & 0,9995604 & 0,9995383 & 0,999679 \\
\cline { 2 - 6 } & $R^{2}$ & 1,0666406 & 1,0349329 & 1,0578678 & 1,05465 \\
\cline { 2 - 6 } & $\left.\mathrm{min}^{-1}\right)$ & 0,0020246 & 0,0026378 & 0,0040263 & 0,003843 \\
\hline \multirow{2}{*}{ Newton e Lewis } & $R^{2}$ & 0,9921441 & 0,9944843 & 0,9878735 & 0,99037 \\
\cline { 2 - 6 } & $R^{2}$ & 0,0018141 & 0,0024967 & 0,0037115 & 0,003566 \\
\hline
\end{tabular}




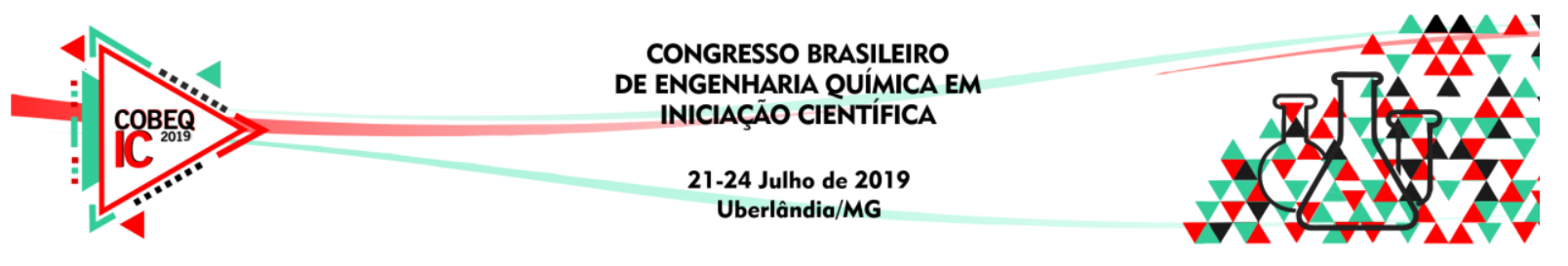

\subsection{Contração Volumétrica}

O índice de contração volumétrica unitária em função do tempo apresentado na Figura 4 ocorreu de forma mais marcante no início da secagem, para todas as temperaturas analisadas. A redução do volume do araçá condiz com outras pesquisas para produtos agrícolas como mamona (Goneli et al., 2011) e cajá-manga (Gouveia et al., 2003). O modelo que apresenta coeficientes de determinação $\left(\mathrm{R}^{2}\right)$ satisfatório é o de Bala \& Woods Adaptado, com média de 0,984, seguido do modelo Polinomial com valores maiores que 0,9712 (Tabela 4). O índice de contração volumétrica da massa em função do teor de água e os valores estimados pelo modelo de Bala \& Woods Adaptado são ressaltados na Figura 5. Os estudos de Fonseca e Cunha (2017) apresentam o comportamento da contração volumétrica similar ao do araçá

Figura 4 - (A) Avaliação da contração volumétrica dos frutos do araçá e (B) ajuste do modelo de Bala \& Woods adaptado nas diferentes temperaturas
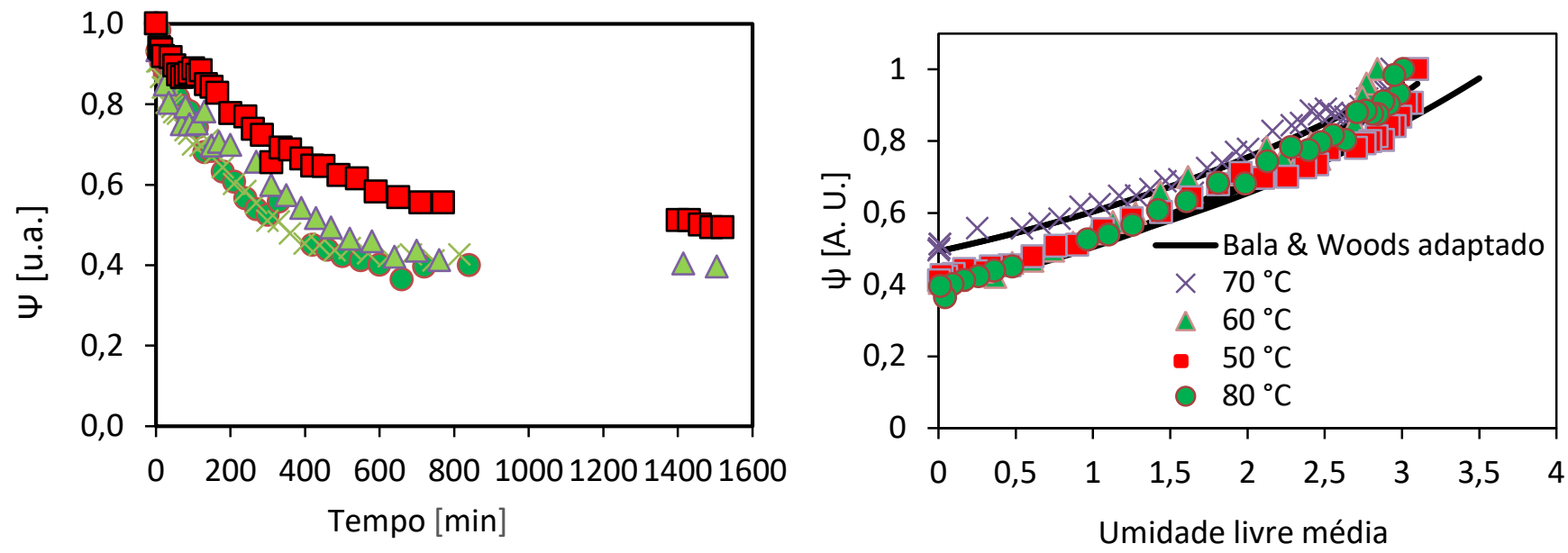

Tabela 4- Parâmetros dos modelos de contração volumétrica para frutos do araçá desidratados em secador de bandejas

\begin{tabular}{|c|c|c|c|c|c|}
\hline Modelos & Parâmetros & $50^{\circ} \mathrm{C}$ & $60^{\circ} \mathrm{C}$ & $70{ }^{\circ} \mathrm{C}$ & $80^{\circ} \mathrm{C}$ \\
\hline \hline \multirow{4}{*}{ Linear adaptado } & $A$ & 0,1058783 & 0,1337183 & 0,1119539 & 0,2843463 \\
\cline { 2 - 6 } & $B$ & 0,0350765 & 0,070462 & 0,0425884 & 0,1468497 \\
\cline { 2 - 6 } & $C$ & 0,3375056 & 0,2187011 & 0,2833481 & 0,0476443 \\
\cline { 2 - 6 } & $R^{2}$ & 0,9717522 & 0,9589248 & 0,9762446 & 0,9720069 \\
\hline \multirow{4}{*}{ Polinomial } & $A$ & 0,4961685 & 0,389845 & 0,4156131 & 0,390002 \\
\cline { 2 - 6 } & $B$ & 0,0802064 & 0,0937409 & 0,0734732 & 0,0797235 \\
\cline { 2 - 6 } & $C$ & 0,0246413 & 0,0298952 & 0,0236282 & 0,0327607 \\
\hline \multirow{3}{*}{$\begin{array}{c}\text { Bala \& Woods } \\
\text { modificado }\end{array}$} & $A$ & 0,9879804 & 0,9711888 & 0,989514 & 0,9881785 \\
\cline { 2 - 6 } & $B$ & $-1270,5996$ & $-1605,4532$ & $-4613,9756$ & $-3193,21$ \\
\cline { 2 - 6 } Bala \& Woods \\
adaptado & $R^{2}$ & 0,0001195 & 0,0001162 & 0,0000403 & 0,0000586 \\
\cline { 2 - 6 } & $A$ & 0,990698 & 0,9586256 & 0,8845683 & 0,9704625 \\
\cline { 2 - 6 } & $C$ & 0,3099076 & 0,3515017 & 0,3312868 & 0,387069614 \\
\cline { 2 - 6 } & $R^{2}$ & 0,4104858 & 0,8089352 & 0,7123509 & 0,8743539 \\
\hline
\end{tabular}




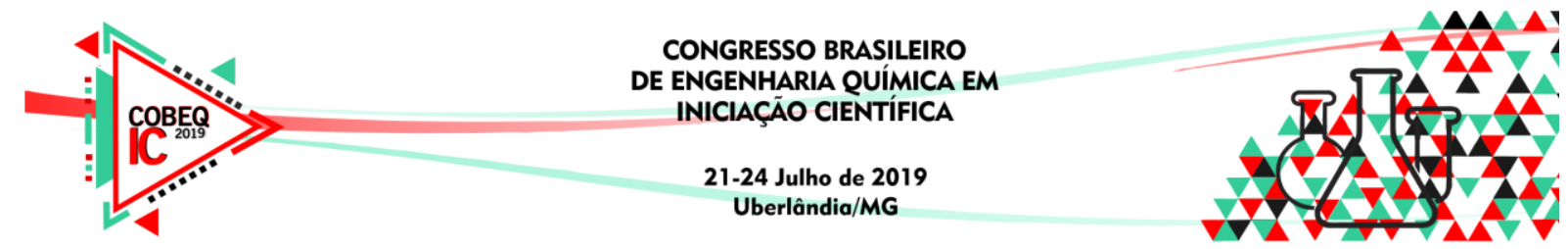

\section{CONCLUSÕES}

A temperatura exerceu influência expressiva sobre a cinética de secagem e a contração volumétrica dos frutos de araçá. O modelo matemático de Midilli et al. (2002) foi o que melhor se ajustou aos dados experimentais da cinética de secagem, com coeficiente de determinação de aproximadamente $99,9 \%$ e para a contração volumétrica foi o modelo de Bala \& Woods adaptado, com coeficiente de determinação de $98,4 \%$.

\section{REFERÊNCIAS}

BROOKER, D. B.; BAKKER-ARKEMA, F. W.; HALL, C. W. Drying and storage of grains and oilseeds. Westport: The AVI Publishing Company, 1992. 450p.

CELESTINO, Sonia Maria Costa. Princípios de Secagem dos Alimentos. Planaltina - DF: Embrapa Cerrados, 2010. 51p.

CORRÊA FILHO, L. C. et al. Cinética de secagem, contração volumétrica e análise da difusão líquida do figo (Ficus carica L.). Revista Brasileira de Engenharia Agrícola e Ambiental, Campina Grande, PB, v. 19, n. 8, p. 797-802, jul. 2014.

CUNHA, Débora Cristina da. Avaliação dos fitoquímicos e das atividades antioxidante celular e antiproliferativa do suco de araçá-una (Psidium Eugeniaefolia) e araçá morango (psidium cattleianum var. lucidum). 2014. 102 f. Dissertação (Mestrado) Curso de Biociências Aplicadas à Saúde, Universidade Federal de Alfenas, Alfenas, 2014.

FONSECA, Kênia Aparecida da; CUNHA, Renata Nepomuceno da. Efeitos da secagem sobre o teor nutricional, a cinética e as alterações morfométricas de sementes de acerola desidratadas em secador de bandejas. 2017. 21 f. TCC (Graduação) - Curso de Engenharia Química, Centro Universitário, Centro Universitário de Patos de Minas, Patos de Minas, 2017.

GONELI, André Luís Duarte et al. Contração volumétrica e forma dos frutos de mamona durante a secagem. Acta Scientiarum. Agronomy, Maringá, v. 33, n. 1, p.1-8. 2011.

GOUVEIA, Josivanda Palmeira Gomes de et al. Determinação de curvas de secagem em frutos do cajá. Revista Brasileira de Produtos Agroindustriais, n.1, p.65-68, 2003.

MIDILLI, A.; KUCUK, H.; YAPAR, Z. A. New model for single-layerdrying. Drying Technology, v.20, p.1503-1513, 2002.

PARK,K.J.;António,G.C.; OLIVEIRA,R.A. Conceitos de processos e equipamentos de secagem. Campinas: Unicamp, 2007.121p.

RESENDE, Osvaldo et al. Forma, tamanho e contração volumétrica do feijão (phaseolus vulgaris 1.) durante a secagem. Revista Brasileira de Produtos Agroindustriais, Campina Grande, v. 7, n. 1, p.15-24, fev. 2005. 